MRS Advances (C) 2016 Materials Research Society

DOI: 10.1557/adv.2016.316

\title{
Human and Environment Influences on Thermoelectric Energy Harvesting Toward Self- Powered Textile-Integrated Wearable Devices
}

\author{
Amanda Myers ${ }^{1}$, Ryan Hodges ${ }^{2}$, and Jesse S. Jur ${ }^{2}$ \\ ${ }^{1}$ Department of Mechanical and Aerospace Engineering, North Carolina State University, 911 \\ Oval Drive, Raleigh, NC 27695, U.S.A. \\ ${ }^{2}$ Department of Textile Engineering, Chemistry and Science, North Carolina State University, \\ 1000 Main Campus Drive, Raleigh, NC 27695, U. S. A.
}

\begin{abstract}
The study of on-body energy harvesting is most often focused on improving and optimizing the energy harvester. However, other factors play a critical factor in the energy harvesting integration techniques of the harvester to close-to body materials of the wearable device. In addition, one must recognize the wide array of human factors and ergonomic factors that lead a variation of the energy harvesting. In this work, key affecting variables at varying onbody locations are investigated for commercial thermoelectric generators (TEGs) integrated within a textile-based wearable platform. For this study, a headband and an armband is demonstrated with five TEGs connected in series in a flexible form factor via Pyralux ${ }^{\circledR}$. These platforms enable comparison of the amount of energy harvested from the forehead versus the upper arm during various external conditions and movement profiles, e.g. running, walking, and stationary for periods of up to 60 minutes. During these tests, ambient temperature, ambient humidity, accelerometry, and instantaneous power are recorded live during the activity and correlated to the energy harvested. Human factors such as skin temperature and application pressure were also analyzed. Our analysis demonstrates that vigorous movement can generate over $100 \mu \mathrm{W}$ of instantaneous power from the headband and up to $35 \mu \mathrm{W}$ from the armband. During the stationary movement profile, the instantaneous power levels of both the headband and the armband decreased to a negligible value. Our studies show that for higher intensities of movement, air convection on the cool side of the TEG is the dominating variable whereas the temperature gradient has a significant effect when the subject is stationary. This work demonstrates key materials and design factors in on-body thermoelectric energy harvesting that allows for a strategic approach to improving the integration of the TEGs.
\end{abstract}

\section{INTRODUCTION}

The focus of on-body energy harvesting is of particular interest due to the sensing and wearability limitations that batteries impose. Eliminating the use of a battery makes the long term adoption of wearable devices more feasible due to improved ease of use and continuous sensing reliability [1]. A study performed by Starner and Paradiso [2] defined a range of power values that can be harvested from the body using various techniques. Notably, the largest amount of energy harvesting potential is found in human motion, or kinetic energy harvesting, through the use of piezoelectric materials. However, the energy harvesting potential drops significantly when movement ceases, yielding an unsteady supply of power to a wearable sensor or gadget [3]-[6]. Thermal energy harvesting offers the most consistent supply of energy for wearable energy harvesting purposes[7]; however, limited temperature differentials between the body and microclimate surrounding it reduce the amount of power that can be produced. Heat spreaders and heat sinks have been used [3], [8]-[11] to aid in maintaining a consistent temperature gradient, but they are rigid and bulky and reduce the comfort of the wearer. 
The location of the energy harvester on the body will determine the efficiency of the energy harvester. For example, a flexible solar panel integrated into a hat will generate much more power than a piezoelectric harvester in the same location. In addition to method of energy harvesting, the on-body location of the wearable defines some design constraints such as form factor, flexibility, materials used, etc. It is crucial to consider the body location of a wearable when designing a novel body-powered sensor or device.

One must consider not only the location of the energy harvester on the body but also what variables will most influence the response of the energy harvester. For a thermal energy generator (TEG), the temperature difference between the surface of the skin and ambient air, air velocity over the TEG, as well as conditions of the ambient air such as humidity could all have an effect on the energy harvesting ability of the TEG regardless of the harvester's location on the body. Additionally, location effects such ass body movement activity and skin temperature can influence the energy harvesting of the TEG.

The goals of this study are to create a flexible, textile-based integration procedure for thermal energy harvesting with commercially available products and then determine which extent by which environmental and physiological variables effect on the response of the TEG. The ultimate goal of the work is to begin the formation of a body map of thermal energy harvesting. In addition, materials considerations are considered that may allow for improved integration of the TEGs to provide improved performance.

\section{METHODS}

The integration of a series of TEGs into a wearable, textile form factor divided into three processes: (i) creating a flexible TEG circuit, (ii) textile preparation and assembly, (iii) system integration. Each of these processes are outlined below.

(i) Creating a flexible TEG circuit. To fabricate a flexible TEG circuit, the TEGs are connected in series on a flexible substrate. This method, outlined in Figure 1(a), uses Pyralux ${ }^{\circledR}$ (Dupont), a flexible, kapton-based substrate that is plated with $\mathrm{Cu}$ on one side. First, the Pyralux ${ }^{\circledR}$ substrate is prepared using a die cutter to create 5 holes, $4.9 \mathrm{~mm}$ x $4.9 \mathrm{~mm}, 6 \mathrm{~mm}$ apart to fit the top header of the TEGs. A mask to define the $\mathrm{Cu}$ interconnects between TEGs is made with the cutter and applied to the Pyralux ${ }^{\circledR}$. The substrate is submerged in Ferric Chloride for 15 $-20 \mathrm{~min}$ to etch away $\mathrm{Cu}$ that is not protected by the mask. The TEGs are placed in series in the cutouts with the phalanges of each TEG maintaining direct contact with a low temperature solder. The substrate with TEGs is then soldered in a reflow oven with the maximum temperature set to $150{ }^{\circ} \mathrm{C}$ for $7 \mathrm{~min}$. The electrical resistance of the 5 TEGs in this configuration is $20 \Omega$.

(ii) Textile preparation and assembly. The headband and armband textile platforms are comprised of $\mathrm{Cu}$ coated taffeta (Less EMF Inc.) to act as a heat spreader, a polyethylene nonwoven (Techtex) for structural support, and a polyester knit (Mood) for the base fabric. The construction of the headband and armband are identical excepting the length and fastener of each. The top of the band is sewn together and a $30 \mathrm{~mm}$ x $125 \mathrm{~mm}$ rectangular cutout is made in the polyester and $\mathrm{Cu}$ taffeta is sewn in its place. The $\mathrm{Cu}$ taffeta has a low resistivity of $0.05 \Omega / \mathrm{sq}$ so it will be an efficient thermal conductor and will extend the effective surface area of the TEG hot side. The polyethylene nonwoven is not only added to provide structural support to the Pyralux ${ }^{\circledR}$ but also to insulate the TEGs and prevent shorting between the Pyralux ${ }^{\circledR}$ and $\mathrm{Cu}$ taffeta. Small holes $\sim 5 \mathrm{~mm} \varnothing$ and $6 \mathrm{~mm}$ apart are punched in the outer polyester layer and in the inner polyethylene layer for TEG insertion. The jersey knit polyester layer is used for extensibility and sweat wicking. The knit structure of the polyester allows for a high level of 
strain which ensures good skin contact with the user through personal size adjustments. The polyester itself is a hydrophobic material and will wick sweat away from the body, aiding in maintaining a temperature difference across the TEGs. A fastener is attached to each band to accommodate size variations of the upper arm and head.

a) Cut holes in Pyralux(R) for TEGs

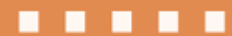

Apply mask to define interconnects

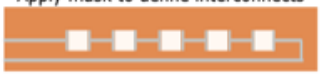

Etch Cu with ferric chloride

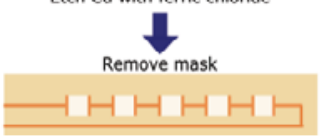

b)

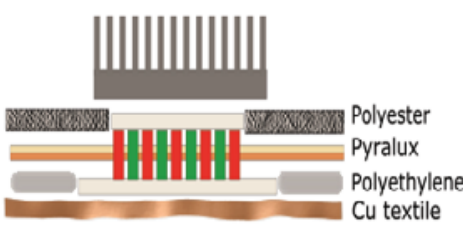

c)

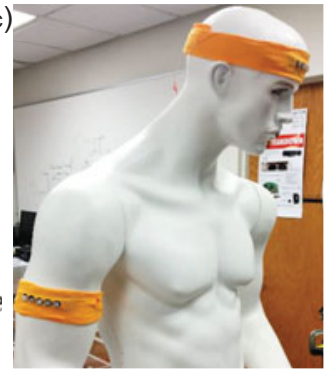

Figure 1: Pyralux interconnect formation schematic (a) Side view of the TEG assembly (b) Assembled armband and headband as worn on a mannequin (c)

iii) System integration. The assembly of the band begins with adhering the TEG/Pyralux ${ }^{\circledR}$ substrate to the inner polyethylene layer with a rubber adhesive (Boots \& Gloves from Shoe Goo). Note, the hot side of the TEG is exposed to ambient air so this part will be exposed through the polyester and polyethylene. Alumina thermal paste (Artic Alumina Thermal Adhesive) is applied to the base of each TEG and the $\mathrm{Cu}$ taffeta is folded over to make contact with each TEG. The addition of thermal paste reduces the chance that insulating air gaps form between the TEG and heat spreader. The band is sewn shut after the glue and paste have cured at room temperature overnight. Small aluminum heat sinks are attached to each TEG header with carbon tape. The final headband and armband can be viewed on a mannequin in Figure 1(c).

\section{PROCEDURE}

The headband and armband were worn by human participants in a trial study to examine the effect of various environmental and movement conditions on thermal energy harvesting. Participants engaged in three monitored activities: i) running/jogging, ii) walking, iii) and sitting while wearing either the headband or armband. The running activity took place outdoors while the walking and sitting activities were performed indoors to simulate typical daily wear of a person. Each activity occurred for at least $15 \mathrm{~min}$ to allow the system to reach a steady state.

An energy harvesting monitoring circuit (EHSS) was developed to collect raw data from the devices as well as to provide additional information about the surrounding environment. The EHSS is equipped with an external temperature sensor, humidity sensor, and an accelerometer in addition to the TEG voltage input and Bluetooth communication. The sampling rate of the TEG input, temperature sensor and humidity sensor is set to $1 \mathrm{~Hz}$ while the accelerometer sampling rate is $100 \mathrm{~Hz}$. To accompany the EHSS, an Android app was developed so the user is able to view the streaming data in real time from the EHSS and head/armband.

\section{DISCUSSION}

It is important to note that the data from the armband and headband were not collected simultaneously as only one EHSS could communicate with the app at a time. The first 
interpretation of the collected data is how the integration of the TEG into a textile platform effects the amount of energy harvested. The data may also be discussed in terms of how external, or environmental, variables effect the amount of energy harvested with the wearable. Before either device was worn, the skin temperature of the forehead and upper arm were taken using a thermal imaging camera, shown in Figure 2. The initial skin temperature of the forehead was recorded as $38.3^{\circ} \mathrm{C}$ and the upper arm showed a skin temperature of $36^{\circ} \mathrm{C}$ while in standard room conditions of $\sim 21^{\circ} \mathrm{C}$.
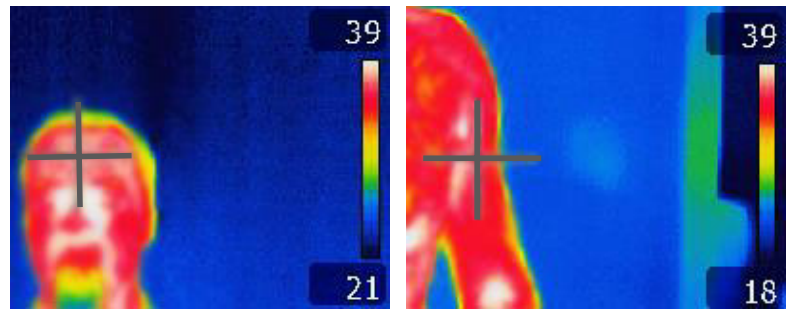

Figure 2: Thermal images of a participant's forehead (left) and upper arm (right). The values were recorded at 38.3 and $36^{\circ} \mathrm{C}$ respectively. Note: the images are intentionally blurred to retain anonymity of the participant.

The instantaneous power, temperature, and aggregate movement, measured from the accelerometer in the $\mathrm{x}$-direction, are shown in the top of Figure 3 with respect to three environmental scenarios: running, walking, and sitting. The temperature and power relationship is highlighted in the leftmost graph, outdoor running; however, dependence of instantaneous power on temperature trends decreases as the user's movement decreases, indicating that the instantaneous power is more a function of induced air flow instead of temperature. To further understand this, one can view the rate of energy harvesting with each movement scenario, shown in the lower graph of Figure 4. The rate of energy harvesting increase can be found by taking the derivative of the total energy harvested over the time period of running, walking, or sitting. The rate of energy harvesting while running is approximately $0.21 \mathrm{~mJ} / \mathrm{s}$ which decreases to $0.13 \mathrm{~mJ} / \mathrm{s}$ while walking before decreasing to $0.001 \mathrm{~mJ} / \mathrm{s}$ while the subject is seated. This difference in energy harvesting rates is attributed to the air convection induced through the heat sinks.

The headband data is comparable to the armband data in that the user movement has a more significant effect on the instantaneous power level than the ambient temperature. However, due to the location, the movement intensity while the user is walking or running is smaller than the armband. A noticeable increase of instantaneous power compared to the armband is observed with the headband, due to a higher skin temperature on the forehead vs. the upper arm as well as better skin contact due to the lack of hair. Although the instantaneous power is higher with the headband, the rate of energy harvesting is significantly lower while running or walking at 0.078 $\mathrm{mJ} / \mathrm{s}$ and $0.044 \mathrm{~mJ} / \mathrm{s}$, respectively, and dropping to $0.003 \mathrm{~mJ} / \mathrm{s}$ when seated.

While the armband has lower instantaneous power levels, its rate of energy harvesting while the user is moving is significantly higher than the headband; indicating that it would harvest more energy over a longer period of time while in motion. The headband shows better promise for energy harvesting while the subject is stationary due to larger temperature gradients 
which yield a faster energy harvesting rate. The results of the thermal energy harvesting body map guide the design and placement of other form factors to meet this requirement.
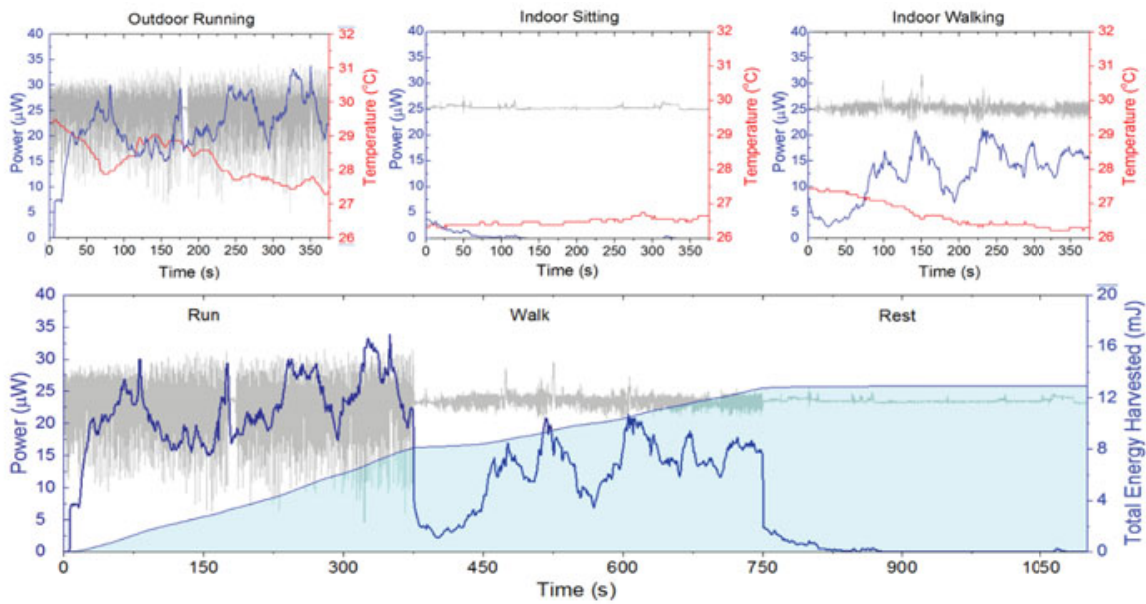

Figure 3: Instantaneous power (blue), temperature (red), and aggregate movement (gray) from the armband are compared with respect to outdoor running and indoor walking and sitting (top left to right). The lower graph shows the rate of energy harvesting with respect to the various movement profiles (bottom).
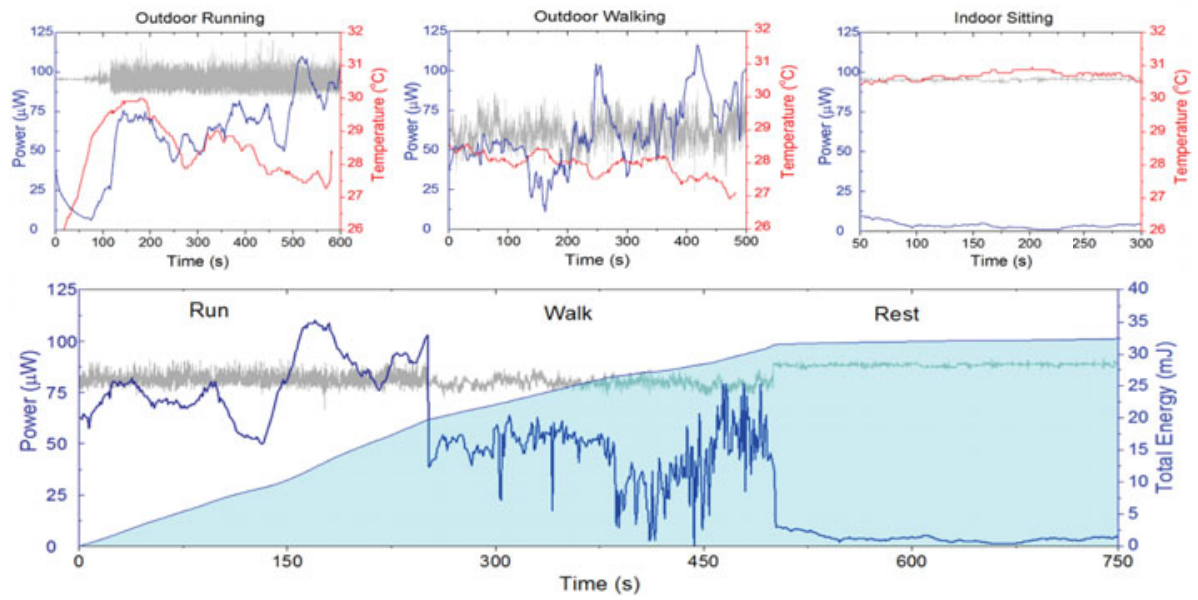

Figure 4: Instantaneous power (blue), temperature (red), and aggregate movement (gray) from the headband are compared with respect to outdoor running and indoor walking and sitting (top 
left to right). The lower graph shows the rate of energy harvesting with respect to the various movement profiles (bottom).

\section{CONCLUSION}

The armband and headband created in this study demonstrate successful integration of TEGs into a wearable textile platform. The Pyralux ${ }^{\circledR}$ facilitates the devices to conform to the body giving the TEGs secure skin contact to reduce the thermal resistance between the skin and TEGs. Testing the devices in various conditions defined the key environmental variables to take into consideration when improving TEG integration. The comparison of on body locations highlighted the need for different integration techniques for various wearable locations. The armband was found to be better suited for an active, mobile user while the headband showed better performance when the user was stationary. These tests created a baseline for which improvements to the TEG integration design can be compared to. Future work will include investigating the textile structure and materials around the TEG to improve the thermal management of the wearable and increase the temperature difference across the TEG.

\section{REFERENCES}

[1] V. Misra, A. Bozkurt, B. Calhoun, T. Jackson, J. Jur, J. Lach, B. Lee, J. Muth, O. Oralkan, M. Ozturk, S. Trolier-McKinstry, D. Vashaee, D. Wentzloff, and Y. Zhu, "Flexible technologies for self-powered wearable health and environmental sensing," Proc. IEEE, vol. 103, no. 4, pp. 665-681, 2015.

[2] T. Starner and J. Paradiso, "Human generated power for mobile electronics," Low-power Electron. Des., vol. 1990, 2004.

[3] V. Leonov and R. J. M. Vullers, "Wearable Thermoelectric Generators for Body-Powered Devices," J. Electron. Mater., vol. 38, no. 7, pp. 1491-1498, Jan. 2009.

[4] A. Harb, "Energy harvesting: State-of-the-art," Renew. Energy, vol. 36, no. 10, pp. 26412654, Oct. 2011.

[5] B. P. D. Mitcheson, M. Ieee, E. M. Yeatman, S. M. Ieee, G. K. Rao, S. M. Ieee, A. S. Holmes, and T. C. Green, "Energy Harvesting From Human and Machine Motion forWireless Electronic Devices,” vol. 96, no. 9, pp. 1457-1486, 2008.

[6] M. Wahbah, M. Alhawari, B. Mohammad, H. Saleh, and M. Ismail, "Characterization of Human Body-Based Thermal and Vibration Energy Harvesting for Wearable Devices," IEEE J. Emerg. Sel. Top. Circuits Syst., vol. 4, no. 3, pp. 354-363, Sep. 2014.

[7] J. W. Stevens, "Optimal design of small $\Delta \mathrm{T}$ thermoelectric generation systems," Energy Convers. Manag., vol. 42, no. 6, pp. 709-720, Apr. 2001.

[8] V. Leonov and R. J. M. Vullers, "Wearable electronics self-powered by using human body heat: The state of the art and the perspective," J. Renew. Sustain. Energy, vol. 1, no. 6, p. $062701,2009$.

[9] V. Leonov, C. Van Hoof, and R. J. M. Vullers, "Thermoelectric and Hybrid Generators in Wearable Devices and Clothes," in 2009 Sixth International Workshop on Wearable and Implantable Body Sensor Networks, 2009, pp. 195-200.

[10] V. Leonov, "Energy Harvesting for Self-Powered Wearable Devices," in Wearable Monitoring Systems, A. Bonfiglio and D. De Rossi, Eds. Boston, MA: Springer US, 2011, pp. $27-49$.

[11] M. Lossec, B. Multon, and H. Ben Ahmed, "Sizing optimization of a thermoelectric generator set with heatsink for harvesting human body heat," Energy Convers. Manag., vol. 68, pp. 260-265, Apr. 2013. 